\title{
Changes in peripheral mitochondrial DNA copy number in metformin-treated women with polycystic ovary syndrome: a longitudinal study
}

Po-Kai Yang ${ }^{1,2}$, Chia-Hong Chou', Chin-Hao Chang ${ }^{3}$, Shee-Uan Chen ${ }^{1,2}$, Hong-Nerng Ho ${ }^{1,2,4}$ and Mei-Jou Chen ${ }^{1,2,5^{*}}$ (i)

\begin{abstract}
Background: Patients with polycystic ovarian syndrome (PCOS) are associated with known alterations in mitochondria DNA copy number (mtDNA-CN). The aim of this study is to study the change in mtDNA-CN in patients with PCOS who were treated with metformin.

Methods: This is a prospective cohort of patients with PCOS, who received metformin for one year. From 2009 to 2015, 88 women diagnosed with PCOS, based on the Rotterdam criteria, were enrolled. Serial measurements of mtDNA-CN, 8-hydroxydeoxyguanosine (8-OHdG), anthropometric, metabolic, endocrine, and inflammatory markers were obtained before and after 3, 6, and 12 months of treatment.

Results: A significant decrease in mtDNA-CN was seen over the course of one year. Other markers, including 8$\mathrm{OHdG}$, testosterone, free androgen index, blood pressure and liver enzymes, also decreased in the same interval. On regression analysis, there was a significant association between the change in mtDNA-CN and serum total testosterone, and no association between mtDNA-CN and metabolic factors.
\end{abstract}

Conclusions: Treatment with metformin is associated with a time-dependent decrease in mtDNA-CN in patients with PCOS who are treated over the course of one year. This may signify a reduction in mitochondria dysfunction. The change in mtDNA-CN corresponds to a similar change in serum total testosterone, and suggests a possible relationship between mtDNA-CN and testosterone.

(Continued on next page)

\footnotetext{
* Correspondence: mjchen04@ntu.edu.tw

'Department of Obstetrics and Gynecology, National Taiwan University

Hospital, No. 8, Chung-Shan South Road, 100 Taipei, Taiwan

${ }^{2}$ College of Medicine, National Taiwan University, Taipei, Taiwan

Full list of author information is available at the end of the article
}

(c) The Author(s). 2020 Open Access This article is licensed under a Creative Commons Attribution 4.0 International License, which permits use, sharing, adaptation, distribution and reproduction in any medium or format, as long as you give appropriate credit to the original author(s) and the source, provide a link to the Creative Commons licence, and indicate if changes were made. The images or other third party material in this article are included in the article's Creative Commons licence, unless indicated otherwise in a credit line to the material. If material is not included in the article's Creative Commons licence and your intended use is not permitted by statutory regulation or exceeds the permitted use, you will need to obtain permission directly from the copyright holder. To view a copy of this licence, visit http://creativecommons.org/licenses/by/4.0/ The Creative Commons Public Domain Dedication waiver (http://creativecommons.org/publicdomain/zero/1.0/) applies to the data made available in this article, unless otherwise stated in a credit line to the data. 
(Continued from previous page)

Trial registration: ClinicalTrials.gov, NCT00172523. Registered September 15, 2005

Keywords: Humans, Female, Polycystic ovary syndrome, Testosterone, Metformin, DNA mitochondrial, 8-Hydroxy-2'Deoxyguanosine

\section{Background}

Polycystic ovary syndrome (PCOS) is a common endocrine disorder in women of reproductive age and is characterized by chronic anovulation, infertility, hyperandrogenism, insulin resistance and metabolic syndrome [1]. In addition to metabolic derangements, there is increased production of reactive oxygen species (ROS) [2]. As oxidative damages from ROS are related to cardiovascular diseases and metabolic syndromes [3], this may explain the high prevalence of hyperlipidemia, hypertension, and steatohepatitis in patients with PCOS $[4,5]$.

Mitochondria functions, which are related to the production of ROS [6], have been assessed in patients with PCOS. Studies on leukocyte-isolated mitochondrion from patients with PCOS have shown reduced oxidative capacity [7], and decreased mitochondria mass [8]. Mitochondria DNA copy number (mtDNA-CN) are also reduced in these patients [9]. Alterations in mtDNA-CN, which may be due to increased mitochondria replication $[10,11]$ or destruction [12], represent functional disturbances of the mitochondria, and have been linked to a number of diseases $[13,14]$. The existance of alterations in mtDNA-CN in patients with PCOS [9] suggests an underlying dysfunction in the mitochondrion of these patients.

Metformin, an insulin sensitizer primarily used in the treatment of type 2 diabetes, has been employed as treatment for patients with PCOS. It has been linked to the correction of several clinical abnormalities, including metabolic, hormonal, ovulatory, and menstrual dysfunctions [15-17]. It also reduces the ROS production from inhibition of complex I in the mitochondria [18-20]. In women with PCOS, metformin has resulted in reduced oxidative markers [21-23], improved mitochondrial respiration capacity, energy utilization, and mitochondrial mass [8]. However, there has been no study on the change in mtDNA-CN in patients with PCOS that were treated with metformin.

This study investigates the change in mtDNA-CN in metformin-treated patients with PCOS over 1 year. Changes in 8-hydroxy-2-deoxyguanosine (8-OHdG), a marker of oxidative DNA damage [24], and metabolic, anthropometric, and hormonal markers were evaluated. The change in mtDNA-CN was evaluated in association with the changes in other clinical markers, to assess for possible interactions. To the best of our knowledge, this is the first human study to evaluate the change in mtDNA-CN in metformin-treated patients.

\section{Methods}

Study subjects

Eighty-eight women with PCOS were enrolled into the study at the National Taiwan University Hospital from 2009 to 2015 . All patients were enrolled from the reproductive endocrinology clinic with complaints of menstrual irregularities or hyperandrogenism. Diagnosis of PCOS was based on the Rotterdam criteria [25]. Other endocrine dysfunction such as hyperprolactinemia, thyroid dysfunction, Cushing syndrome, congenital adrenal hyperplasia, adrenal tumors, and virilizing tumors were excluded. Patients medicated with hormones, chemotherapy or immunosuppressive agents in the past 6 months were excluded. All major systemic diseases, including autoimmune diseases, central nervous system diseases, and malignancy were excluded. The Institutional Review Board of the National Taiwan University Hospital approved this study. Signed informed consents were obtained from all patients or their legal guardians before data collection.

\section{Protocol and data collection}

Patients received evaluation for hormonal, metabolic, anthropometric, hepatic, inflammatory, oxidative markers and blood pressures at baseline (M0). Body mass index (BMI) was calculated as weight $(\mathrm{kg})$ divided by squared height $\left(\mathrm{m}^{2}\right)$. Overweight was defined as a BMI greater than $23 \mathrm{~kg} / \mathrm{m}^{2}$, based on the WHO recommendations for Asians [26], and abdominally obese was defined as a waist circumference $\geq 80 \mathrm{~cm}$ [27]. Systolic (SBP) and diastolic (DBP) blood pressure were calculated as the mean of two measurements. The descriptions for blood collections have been detailed previously [28]. Briefly, the blood samples were collected after overnight fasts, and in the early follicular phase in women with spontaneous menses and immediately before hormone-induced withdrawal bleeding in amenorrheic women. A standard $75 \mathrm{~g}$ oral glucose test was performed at baseline, and patients were classified as normal, glucose impaired, or diabetic, based on the 2-h blood glucose cutoffs of 140 $\mathrm{mg} / \mathrm{dL}$ and $200 \mathrm{mg} / \mathrm{dL}$ [29]. 
Metformin (Loditon, Standard Chem and Pharm, Taiwan) was started at $500 \mathrm{mg} /$ day in the first month, $1000 \mathrm{mg} /$ day in the second month, $1500 \mathrm{mg} /$ day in the third month, and continued at $1500 \mathrm{mg} /$ day for one year, as described previously [17]. A decrease to $1000 \mathrm{mg}$ daily dose was allowed for patients who reported gastrointestinal symptoms, such as abdominal distention and cramping, that affected daily activities. Any lowering of doses was recorded as having received a non-maximal regimen. Patients returned for prescriptions every month in the first 6 months, and every 2 months in the last 6 months. Compliance was assessed at every prescription for the preceding interval, based on the patients' report of retaining missed pills from that interval. The number of intervals with missed pills was normalized by the number of intervals with a prescription, and a rate $<30 \%$ was considered compliant. Patients were reevaluated for hormonal, metabolic, anthropometric, hepatic, inflammatory, oxidative markers and blood pressures after 3 (M3), 6 (M6), and 12 months (M12) of treatment.

\section{Assay methods}

Plasma glucose, glutamic oxaloacetic transaminase (GOT), glutamic pyruvic transaminase (GPT), and high sensitivity C-reactive protein (hsCRP) were measured using an autoanalyzer (Toshiba TBA-120 FR; Toshiba, Tokyo, Japan). Serum insulin levels were determined using a microparticle enzyme immunoassay in an AxSYM system (Abbott Laboratories, Dainabot Co., Tokyo, Japan). Serum total testosterone was measured using radioimmunoassays (RIA; Diagnostic Systems Laboratories, Webster, TX, USA), while serum sex hormone-binding globulin (SHBG) was measured using electrochemiluminescence (Elecsys 2010; Roche Diagnostics, Mannheim, Germany). The free androgen index (FAI) was calculated as FAI $=$ total testosterone $/$ SHBG $\times$ $100 \%$. The homeostatic model assessment-insulin resistance (HOMA-IR) was calculated as HOMA$\mathrm{IR}=($ glucose $(\mathrm{mg} / \mathrm{dl}) \times 0.05551) \times$ insulin $(\mathrm{IU} / \mathrm{ml}) / 22.5$ [30].

Plasma 8-OHdG was assessed using the HT 8-oxo-dG ELISA Kit II (R\&D Systems, Inc., Minneapolis, MN, USA; catalog no. 4380-096-K). Peripheral blood leukocyte DNA was extracted from the buffy coat using a QIAamp DNA kit (Qiagen, Valencia, CA, USA). The mtDNA-CN was assessed using quantitative PCR (SYBR green system, Applied Biosystems, Waltham, MA, USA) according to a previous study [31]. The amount of mtDNA-CN was normalized to the expression of nuclear beta-globin. Primers for the mitochondrial ND1 gene were 5' $^{\prime}$-AACATACCCATGGCCAACCT-3' and 5'AGCGAAGGGTTGTAGTAGCCC-3'. Primers for the nuclear beta-globin gene were 5' -GAAGAGCCAAGGACAGGTAC-3' and 5'-CAACTTCATCCACGTTCACC-
3 '. The threshold cycle number $(\mathrm{Ct})$ was defined as the number of polymerase chain reaction cycles needed to produce $20 \mathrm{ng}$ of DNA product; mtDNA-CN was calculated using the equation: relative copy number $=2^{\Delta \mathrm{Ct}}$ $\left(\Delta \mathrm{Ct}=\mathrm{Ct}_{\text {beta-globin }}-\mathrm{Ct}_{\mathrm{ND1}}\right)$.

\section{Statistical analysis}

Baseline comparisons between groups were performed using the Mann-Whitney $U$ test, and the chi-squared test as appropriate. Correlations at baseline were performed using the Spearman's rank correlation test. The significance of changes in clinical variables at M3, M6, and M12 were compared to the baseline using the paired samples Wilcoxon signed-rank test. The changes in clinical variables with respect to time (in months) under treatment were assessed using the generalized estimating equations (GEE), which are hierarchical regression models that account for repeated measurements [32]. Regressions between the change in mtDNA-CN and changes in other clinical variables were performed using the bivariate GEE model, after normalizing each variable by interval. The normalization step transforms each variable into the logarithm of the magnitude of change in each interval, before modeling with GEE. As GEE is able to model longitudinal data with intermittent missing data, all available data were used for analysis. A twosided significance level of $<0.05$ was considered significant. The data analysis of this study was done in SPSS (SPSS Inc. Released 2008. SPSS Statistics for Windows, Version 17.0. Chicago: SPSS Inc.).

\section{Results}

Baseline characteristics of the patients are summarized in Table 1 . The median age at enrollment was 24 years old with a median BMI of $23.2 \mathrm{~kg} / \mathrm{m}^{2}$, and a median waist circumference of $84 \mathrm{~cm}$. Fifty-one percent of the patients were considered overweight, and $64.8 \%$ were abdominally obese. The median fasting glucose was 82.0 $\mathrm{mg} / \mathrm{dL}$, and the median HOMA-IR was 0.8. Metabolically, none of the patients had overt diabetes on the $2-\mathrm{h}$ $75 \mathrm{~g}$ glucose tolerance test, and $14.8 \%$ were considered glucose impaired. The patients had a median FAI of $2 \%$, and a LH/FSH ratio of 1.7. Medication parameters show that $46.4 \%$ of patients received a non-maximal regimen, and $61.9 \%$ of patients were considered compliant.

A stratified analysis based on a BMI cutoff of $\geq 23 \mathrm{~kg}$ / $\mathrm{m}^{2}$ shows that a subgroup with relative insulin resistance could be effectively identified. The overweight group was associated with greater waist size, higher fasting glucose, higher fasting insulin, and higher blood glucose on the 2-h $75 \mathrm{~g}$ glucose tolerance test. The overweight group also had greater percentages of abdominally obese and glucose impairment. Weight appears to affect whether or not patients received a non-maximal regimen, with 
Table 1 Characteristics of the patients in the cohort

\begin{tabular}{|c|c|c|c|c|}
\hline Baseline Parameters & $\begin{array}{l}\text { Total } \\
(\boldsymbol{N}=88)\end{array}$ & $\begin{array}{l}\text { Normal Weight } \\
(\boldsymbol{N}=43)\end{array}$ & $\begin{array}{l}\text { Overweight } \\
(\boldsymbol{N}=45)\end{array}$ & $\boldsymbol{p}$-value between weights \\
\hline Age (years) & $24.0(20.0-28.0)$ & $24.0(21.0-26.8)$ & $25.5(17.3-29.8)$ & 0.697 \\
\hline BMl $\left(\mathrm{kg} / \mathrm{m}^{2}\right)$ & $23.2(20.6-29.8)$ & $20.9(19.2-21.9)$ & $30.6(25.4-37.4)$ & $<0.001^{*}$ \\
\hline Overweight & $51.1 \%(45 / 88)$ & $0 \%(0 / 43)$ & $100 \%(45 / 45)$ & - \\
\hline Waist (cm) & $84.0(77.0-94.0)$ & $76.3(73.6-81.5)$ & $93.8(86.6-109.0)$ & $<0.001^{*}$ \\
\hline Abdominally obese & $64.8 \%(57 / 88)$ & $32.6 \%(14 / 43)$ & $95.6 \%(43 / 45)$ & $<0.001^{*}$ \\
\hline Fasting glucose (mg/dL) & $82.0(78.0-86.8)$ & $80.5(76.0-84.8)$ & $87.0(81.3-91.0)$ & $<0.001^{*}$ \\
\hline Fasting insulin (mIU/L) & $4.3(2.0-12.9)$ & $2.0(2.0-4.7)$ & $13.8(6.5-21.4)$ & $<0.001^{*}$ \\
\hline HOMA-IR & $0.8(0.4-2.7)$ & $0.4(0.4-1.0)$ & $2.9(1.3-4.9)$ & $<0.001^{*}$ \\
\hline 75 g GTT 2 h (mg/dL) & $105.0(85.8-129.0)$ & $91.5(80.3-104.3)$ & $122.5(115.3-142.3)$ & $<0.001^{*}$ \\
\hline Glucose impaired & $14.8 \%(8 / 54)$ & $0.0 \%(0 / 28)$ & $30.7 \%(8 / 26)$ & $0.001^{*}$ \\
\hline Testosterone (ng/ml) & $0.6(0.4-0.8)$ & $0.5(0.3-0.7)$ & $0.6(0.4-0.9)$ & 0.676 \\
\hline SHBG $(\mathrm{nmol} / \mathrm{l})$ & $31.1(19.0-45.0)$ & $42.2(34.4-59.4)$ & $18.2(15.2-25.3)$ & $<0.001^{*}$ \\
\hline FAl (\%) & $2.0(1.2-3.4)$ & $1.1(0.8-1.9)$ & $3.5(2.2-4.4)$ & $<0.001^{*}$ \\
\hline $\mathrm{FSH}(\mathrm{mlU} / \mathrm{ml})$ & $6.1(5.3-7.4)$ & $6.3(5.5-7.8)$ & $5.6(5.1-6.6)$ & $0.037^{*}$ \\
\hline LH (mlU/ml) & $10.8(7.7-14.9)$ & $13.8(10.3-16.7)$ & $9.1(6.4-10.8)$ & $<0.001^{*}$ \\
\hline LH/FSH ratio & $1.7(1.2-2.5)$ & $2.1(1.8-2.7)$ & $1.5(1.2-1.8)$ & $0.001^{*}$ \\
\hline $\mathrm{GOT}(\mathrm{U} / \mathrm{L})$ & $19.0(17.0-23.0)$ & $18.5(16.0-21.8)$ & $20.5(19.0-31.3)$ & 0.464 \\
\hline GPT (U/L) & $17.0(12.0-24.0)$ & $13.0(11.0-17.8)$ & $24.0(16.0-38.3)$ & $<0.001^{*}$ \\
\hline hsCRP (mg/dL) & $0.09(0.03-0.27)$ & $0.04(0.02-0.08)$ & $0.31(0.09-0.63)$ & $<0.001^{*}$ \\
\hline $8-\mathrm{OHdG}$ & $34.6(27.7-45.4)$ & $38.5(30.6-53.9)$ & $31.0(25.3-42.6)$ & 0.210 \\
\hline mtDNA-CN & $55.4(26.1-100.3)$ & $47.2(24.8-77.5)$ & $50.7(23.9-98.6)$ & 0.786 \\
\hline \multicolumn{5}{|l|}{ Medication Parameters } \\
\hline Non-maximal regimen & $46.4 \%(39 / 84)$ & $64.3 \%(27 / 42)$ & $28.6 \%(12 / 42)$ & $0.001^{*}$ \\
\hline Compliant & $61.9 \%(52 / 84)$ & $61.9 \%(26 / 42)$ & $61.9 \%(26 / 42)$ & 0.999 \\
\hline
\end{tabular}

Data given as median (Q1-Q3); p-value < 0.05 is denoted with * for comparisons between normal-weight and overweight groups; BMI Body mass index, HOMA-IR Homeostatic model assessment-insulin resistance, GTT Glucose tolerance test, SHBG Sex hormone binding globulin, FAI Free androgen index, FSH Follicle stimulating hormone, LH Luteinizing hormone, GOT/GPT Glutamic oxaloacetic/pyruvic transaminase, hsCRP High sensitivity C-reactive protein, 8-OHdG 8-hydroxy-2deoxyguanosine, mtDNA-CN Mitochondrial DNA copy number

$64.3 \%$ of normal weight patients and $28.6 \%$ of overweight patients having received a lower dose than originally specified $(p=0.003)$. Weight did not affect whether patients were compliant with the prescribed regimen.

At study baseline, mtDNA-CN did not correlate with any of the anthropometric measures, inflammatory markers, metabolic markers, nor with $8-\mathrm{OHdG}$ levels (Table 2). There was no correlation between 8-OHdG and age, BMI, waist circumference, testosterone, SHBG, FAI, fasting insulin, HOMA-IR, 2-h 75 g glucose tolerance test, GOT, SBP, DBP, hsCRP, and mtDNA-CN. There was a negative correlation between fasting glucose and 8-OHdG $(\mathrm{r}=-0.25524, p=0.017)$, and between GPT and 8-OHdG ( $\mathrm{r}=-0.23377, p=0.029)$.

Over the course of the study, 88 women completed workup at baseline, 85 at 3 months, 87 at 6 months, and 61 at 12 months. Treatment with metformin was associated with significant time-dependent decreases in testosterone, SHBG, FAI, GOT, GPT, SBP, DBP, mtDNA-CN, and 8-OHdG (Table 3). When compared pair-wise with the baseline, BMI, testosterone, FAI and 8-OHdG showed decreases at M3, M6, and M12. Significant decreases were seen in GOT, GPT, DBP, and mtDNA-CN at M6 and M12. SHBG, SBP, and HSCRP were significantly decreased at M12, while fasting glucose and HOMA-IR were decreased at M6 and M3, respectively. No significant change was seen in fasting insulin.

A number of clinical variables were evaluated as possible explanatory factors for the change in mtDNA-CN. Regressions of the change in mtDNA-CN due to changes in other clinical variables were performed. Betweenvariables comparisons were conducted on normalized values calculated as the logarithm of the magnitude of change (Fig. 1). These transformed values are negative for decreases and positive for increases, and are 
Table 2 Correlations between mtDNA-CN, 8-OHdG, and clinical variables at baseline

\begin{tabular}{lll}
\hline & mtDNA-CN & 8-OHdG \\
\hline Age & $-0.06283(0.565)$ & $-0.07736(0.476)$ \\
BMl & $-0.03618(0.741)$ & $-0.10975(0.312)$ \\
Waist circumference & $-0.02719(0.804)$ & $-0.04646(0.669)$ \\
Testosterone & $0.18848(0.082)$ & $0.10680(0.325)$ \\
SHBG & $0.02572(0.816)$ & $0.17169(0.116)$ \\
FAl & $0.12249(0.267)$ & $-0.11352(0.301)$ \\
Fasting glucose & $0.07002(0.522)$ & $-0.25524(0.017)^{*}$ \\
Fasting insulin & $0.02663(0.808)$ & $-0.02919(0.788)$ \\
HOMA-IR & $0.00987(0.928)$ & $-0.07668(0.480)$ \\
75 g GTT 2h & $0.14033(0.312)$ & $-0.06693(0.634)$ \\
GOT & $-0.01371(0.900)$ & $-0.15375(0.155)$ \\
GPT & $-0.08597(0.431)$ & $-0.23377(0.029)^{*}$ \\
SBP & $-0.02479(0.821)$ & $0.01901(0.861)$ \\
DBP & $-0.09383(0.390)$ & $-0.00817(0.940)$ \\
hsCRP & $-0.12933(0.290)$ & $-0.06189(0.608)$ \\
8-OHdG & $0.06776(0.538)$ & - \\
mtDNA-CN & - & $0.06776(0.538)$ \\
\hline Data given asr & &
\end{tabular}

Data given as $\mathrm{r}$ ( $\mathrm{p}$-value); $\mathrm{p}$-value $<0.05$ is denoted with *; BMI Body mass index, SHBG Sex hormone binding globulin, FAl Free androgen index, HOMA-IR Homeostatic model assessment-insulin resistance, GTT Glucose tolerance test, GOT/GPT Glutamic oxaloacetic/pyruvic transaminase, SBP Systolic blood pressure, $D B P$ Diastolic blood pressure, $h s C R P$ High sensitivity C-reactive protein, 8-OHdG 8-hydroxy-2-deoxyguanosine, mtDNA-CN Mitochondrial DNA copy number summarized in the supplement (See Supplementary Table 1, Additional file 1). The strength of the relationships were represented by the coefficient estimates of the explanatory variables in the GEE models (Table 4). From the GEE models, the change in mtDNA-CN appeared unrelated to the changes in BMI, SHBG, FAI, fasting glucose, fasting insulin, HOMA-IR, GOT, GPT, SBP, DBP, hsCRP, or 8-OHdG. There was a sole statistical relationship between the change in mtDNA-CN and the change in testosterone $(\beta=0.3236, p=0.0357)$.

The change in mtDNA-CN due to other patient factors, such as overweight, abdominally obese, glucose impaired, non-maximal regimen, and compliant status was examined. The change in mtDNA-CN was unrelated to any of these factors (Table 4). We also tested whether compliance affected the changes in other clinical variables (See Supplementary Table 2, Additional file 1). There were no relations between compliance and the change in any of the clinicial variables.

From the longitudinal plots of the variables (Fig. 1), a few variable pairs appeared to have similar changes in median values over time, including GOT/GPT, SBP/ DBP, HOMA-IR/hsCRP, and 8-OHdG/SHBG pairs. We assessed for associations between these pairs using the GEE models. The relationships between GOT and GPT $(\beta=0.5131, p<0.0001)$, and between SBP and DBP $(\beta=$ $0.0418, p<0.0001)$ were significant, while the relationships between HOMA-IR and hsCRP, and between 8OHdG and SHBG were not. This perceived discrepancy between the GEE results and the visual interpretation of the longitudinal plots can be explained by the

Table 3 The change in median values for each variable during the one-year treatment with metformin

\begin{tabular}{|c|c|c|c|c|c|c|}
\hline & Baseline $(N=88)$ & 3 months $(N=85)$ & 6 months $(N=87)$ & 12 months $(N=61)$ & Time Estimate (months) & $p$-value \\
\hline BMl $\left(\mathrm{kg} / \mathrm{m}^{2}\right)$ & $23.2(20.6-29.8)$ & $22.7^{*}(20.7-29.3)$ & $22.7^{*}(20.7-29.0)$ & $22.6^{*}(20.5-29.0)$ & -0.048 & 0.123 \\
\hline Testosterone (ng/ml) & $0.61(0.41-0.81)$ & $0.48^{*}(0.33-0.68)$ & $0.40 *(0.26-0.60)$ & $0.36^{*}(0.28-0.52)$ & -0.019 & $<0.001^{* *}$ \\
\hline SHBG (nmol/l) & $31.1(19.0-45.0)$ & $30.5(20.0-46.6)$ & $32.4(18.8-46.0)$ & $17.3^{*}(17.7-36.7)$ & -0.323 & $0.014^{* *}$ \\
\hline $\mathrm{FAl}(\%)$ & $6.84(4.07-11.86)$ & $5.05^{*}(3.53-8.34)$ & $4.27^{*}(2.52-8.34)$ & $5.06^{*}(3.10-7.53)$ & -0.178 & $<0.001^{* *}$ \\
\hline Fasting glucose (mg/dL) & $82(78-87)$ & $82(77-85)$ & $81^{*}(78-85)$ & $84(79-87)$ & 0.046 & 0.437 \\
\hline Fasting insulin (mlU/L) & $4.27(2.00-12.93)$ & $3.70(2.00-11.25)$ & $4.97(2.00-9.79)$ & $4.28(2.00-9.04)$ & -0.137 & 0.083 \\
\hline HOMA-IR & $0.84(0.41-2.68)$ & $0.76^{*}(0.39-2.28)$ & $0.95(0.40-2.07)$ & $0.75(0.41-1.84)$ & -0.029 & 0.103 \\
\hline GOT(U/L) & $19(17-23)$ & $18(15-23)$ & $17^{*}(15-21)$ & $16^{*}(13-20)$ & -0.498 & $0.002^{* *}$ \\
\hline GPT (U/L) & $17(12-24)$ & $17(12-29)$ & $15^{*}(10-23)$ & $12^{*}(9-18)$ & -0.739 & $0.012^{* *}$ \\
\hline SBP $(\mathrm{mmHg})$ & $112(105-124)$ & $112(104-122)$ & $112(105-122)$ & $109 *(101-118)$ & -0.251 & $0.010^{* *}$ \\
\hline $\mathrm{DBP}(\mathrm{mmHg})$ & $72(66-78)$ & $72(65-79)$ & $71 *(65-79)$ & $70^{*}(63-77)$ & -0.274 & $<0.001^{* *}$ \\
\hline hsCRP (mg/dL) & $0.088(0.038-0.287)$ & $0.099(0.015-0.262)$ & $0.093(0.028-0.283)$ & $0.062^{*}(0.026-0.252)$ & -0.006 & 0.267 \\
\hline mtDNA-CN & $55.38(26.14-100.27)$ & 34.78 (19.27-78.08) & $33.42^{*}(20.45-55.78)$ & $24.11^{*}(16.92-66.75)$ & -4.509 & $0.003^{* *}$ \\
\hline $8-\mathrm{OHdG}$ & $34.62(27.70-45.44)$ & $31.95^{*}(23.07-42.12)$ & $29.96^{*}(21.21-39.09)$ & $18.57^{*}(12.13-31.15)$ & -1.299 & $<0.001^{* *}$ \\
\hline
\end{tabular}



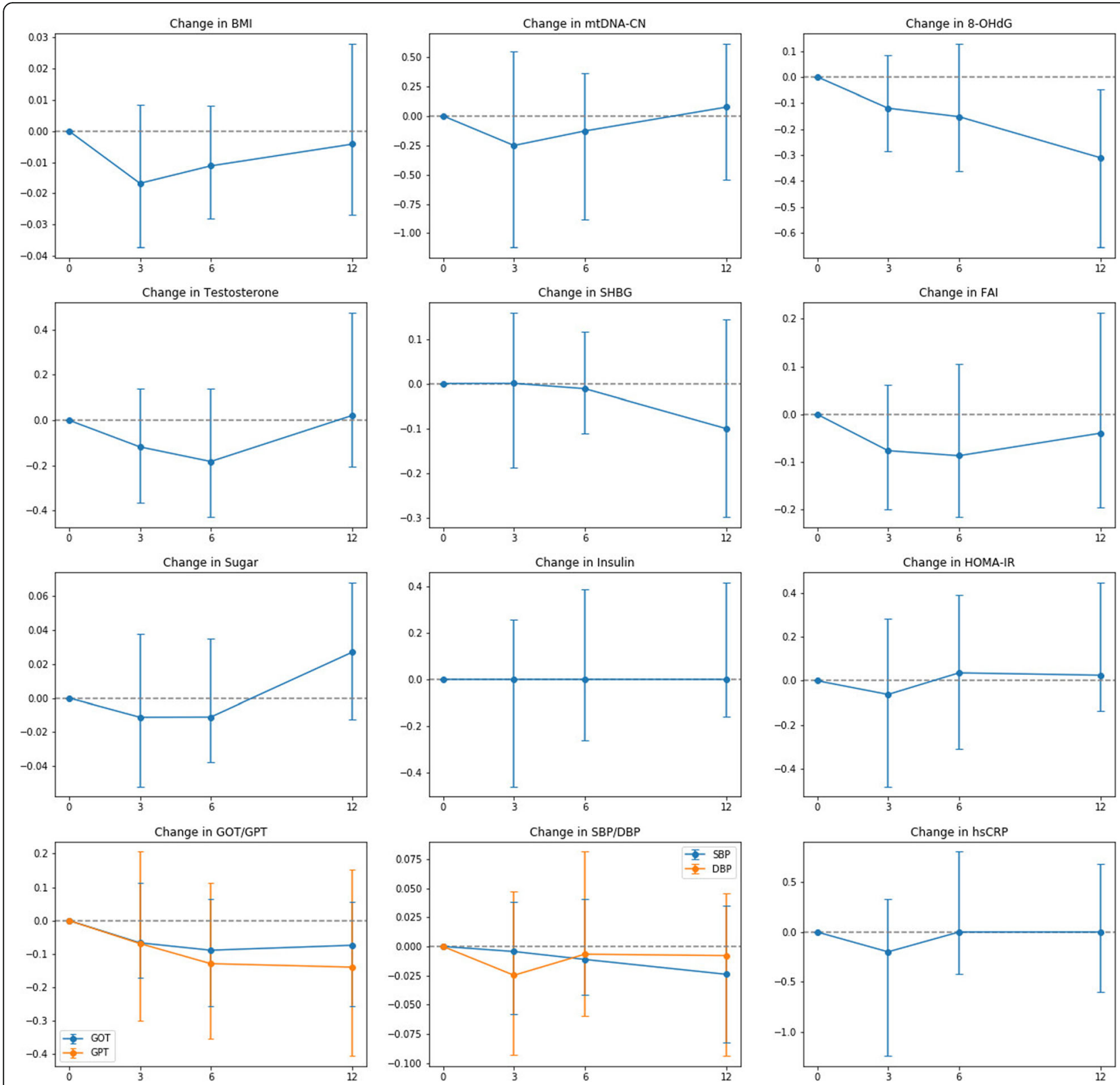

Fig. 1 The normalized median values for the change in clinical variables during treatment. The values representing change were normalized by taking the logarithm of the magnitude of change in each interval. Transformed values are zero for no change, positive values for increases, and negative values for decreases. The points on the line represent the medians, and Q1 and Q3 are denoted by the error bars. Abbreviations are as follows: BMI: Body mass index; SHBG: sex hormone binding globulin; FAl: Free androgen index; HOMA-IR: homeostatic model assessment-insulin resistance; GOT/GPT: glutamic oxaloacetic/pyruvic transaminase; hsCRP: high sensitivity C-reactive protein; SBP: systolic blood pressure; DBP: diastolic blood pressure; mtDNA-CN: mitochondrial DNA copy number; 8-OHdG: 8-hydroxy-2-deoxyguanosine

contribution of subject-level interactions, which are tested by the GEE models. In essence, although the changes in HOMA-IR/hsCRP and 8-OHdG/SHBG pairs may seem similar, judging by sample medians, this may not reflect real changes in the individual. The changes in HOMA-IR/hsCRP and 8-OHdG/SHBG pairs were not statistically significant by this assessment.

\section{Discussion}

As an insulin sensitizer, metformin was originally used to treat insulin resistance associated with PCOS [15]. Since then, the drug has been extensively studied, and has shown activity against many clinical derangements in patients with PCOS, ranging from obesity, hypertension to metabolic, endocrine and menstrual dysfunctions 
Table 4 Coefficient estimates for each clinical variable in predicting change in mtDNA-CN

\begin{tabular}{|c|c|c|c|c|c|}
\hline \multirow{2}{*}{$\begin{array}{l}\text { Longitudinal Variables } \\
\text { BMI }\end{array}$} & \multirow{2}{*}{$\begin{array}{l}\text { Estimate } \\
-2.4737\end{array}$} & \multirow{2}{*}{$\begin{array}{l}\text { SE } \\
1.8465\end{array}$} & \multicolumn{2}{|l|}{$95 \% \mathrm{Cl}$} & \multirow{2}{*}{$\begin{array}{l}\text { p-value } \\
0.1803\end{array}$} \\
\hline & & & -6.0927 & 1.1453 & \\
\hline Testosterone & 0.3236 & 0.1541 & 0.0216 & 4.4097 & $0.0357^{*}$ \\
\hline SHBG & 0.3269 & 0.2903 & -0.2420 & 0.8959 & 0.2600 \\
\hline FAl & 0.3530 & 0.3511 & -0.3352 & 1.0412 & 0.3147 \\
\hline Fasting glucose & 1.5686 & 1.5010 & -1.3734 & 4.5106 & 0.2960 \\
\hline Fasting insulin & 0.0296 & 0.1470 & -0.2584 & 0.3177 & 0.8403 \\
\hline HOMA-IR & 0.0427 & 0.1423 & -0.2362 & 0.3217 & 0.7641 \\
\hline GOT & 0.0387 & 0.2007 & -0.3548 & 0.4322 & 0.8471 \\
\hline GPT & -0.1001 & 0.1347 & -0.3642 & 0.1640 & 0.4575 \\
\hline SBP & -0.9090 & 1.1083 & -3.0813 & 1.2633 & 0.4121 \\
\hline DBP & 0.0612 & 0.7854 & -1.4780 & 1.6005 & 0.9379 \\
\hline hsCRP & -0.0175 & 0.0466 & -0.1089 & 0.0738 & 0.7070 \\
\hline 8-OHdG & -0.1829 & 0.1041 & -0.3869 & 0.0211 & 0.0789 \\
\hline \multicolumn{6}{|l|}{ Baseline Variables } \\
\hline Overweight & 0.0583 & 0.1227 & -0.1822 & 0.2988 & 0.6345 \\
\hline Abdominal obesity & 0.0384 & 0.1392 & -0.2343 & 0.3111 & 0.7826 \\
\hline Glucose impairment & 0.2663 & 0.1842 & -0.0948 & 0.6275 & 0.1483 \\
\hline \multicolumn{6}{|l|}{ Medication Variables } \\
\hline Non-maximal dose & -0.0421 & 0.1241 & -0.2853 & 0.2012 & 0.7346 \\
\hline Compliant & 0.1022 & 0.1300 & -0.1526 & 0.3570 & 0.4318 \\
\hline
\end{tabular}

p-value $<0.05$ is denoted with*; BMI Body mass index, SHBG Sex hormone binding globulin, FAI Free androgen index, HOMA-IR Homeostatic model assessment-insulin resistance, GOT/GPT Glutamic oxaloacetic/pyruvic transaminase, SBP Systolic blood pressure, DBP Diastolic blood pressure, $h s C R P$ High sensitivity C-reactive protein, 8-OHdG 8-hydroxy-2-deoxyguanosine

$[16,17]$. Metformin has also shown activity in reducing oxidative end-products, including malondialdehyde and 8 -OHdG [22, 23]. As the production of oxidative endproducts is intimately tied to mitochondrial functions [6], the effects of metformin on mitochondria functions were explored in one study [8]. In the study, metformin improved mitochondrial respiration capacity, energy utilization, and mitochondria mass in women with PCOS [8]. However, the study provided no data on the change in mtDNA-CN, which has been frequently utilized as a marker of mitochondria dysfunction [33, 34].

This study evaluates the longitudinal change in mtDNA-CN in women with PCOS, who were treated with metformin. In this study, a progressive, timedependent decrease in mtDNA-CN was observed in patients treated with metformin. However, the nature of this decrease in mtDNA-CN is not immediately clear, as the literature diverges on the relationship between mtDNA-CN and diseases. In cross-sectional studies, mtDNA-CN was lower in diseases such as multiple sclerosis and Parkinson's disease [13, 14], while others have found lower levels of mtDNA-CN in normal pregnancies, when compared with pregnancies complicated with gestational diabetes [35]. Although no conclusion can be drawn on the role of mtDNA-CN from these studies, mtDNA-CN tends to correspond with changes in oxidative markers, such as 8-OHdG [35-37]. As 8OHdG is produced during ROS-mediated DNA damages, it is often utilized as a marker of intra-cellular oxidative stress [24]. Due to its associations with 8-OHdG, many studies have similarly ascribed a decrease in mtDNA-CN to a decrease in cellular stress and damages $[10,11,38]$.

In the absence of an authoritative study on the nature of these mtDNA-CN changes, the difficulty lies in reconciling the decrease in mtDNA-CN associated with PCOS [9], and the decrease in mtDNA-CN associated with metformin treatment in these same patients. In both situations, however, 8-OHdG appears to corroborate with the change in mtDNA-CN. Both 8-OHdG and mtDNA$\mathrm{CN}$ are lower in patients with PCOS $[9,39]$, and lower with metformin treatment [23]. As no causal research is currently available, any explanation is speculative, but these correlations do point to an improved oxidative state. The means through which 8-OHdG and mtDNA$\mathrm{CN}$ are reduced in PCOS is unknown. In the absence of studies on the nature of these mtDNA-CN changes, it may be prudent for clinicians to remain wary of possible adverse effects from metformin on energy metabolism in these patients.

In this study, we also examined if a statistical relationship can be established between mtDNA-CN and 8-OHdG. When assessed with regression, the change in 8-OHdG did not contribute significantly to the change in mtDNA-CN. This shows that although the markers are linked clinically, they are not correlated when assessed in a measurement-by-measurement basis. In fact, mtDNA-CN and 8-OHdG differed in their associations with patient characteristics. At study baseline, mtDNA-CN was uncorrelated with metabolic factors, while 8-OHdG was correlated with fasting glucose and GPT. The significant association with GPT and not with GOT may be explained by the greater sensitivity of GPT for metabolic syndrome [40], which also affects 8-OHdG [41].

The change in mtDNA-CN was also uncorrelated with most other clinical variables, including BMI, which was implicated in previous studies $[9,42]$. Only testosterone appears to be statistically related to the change in mtDNA-CN, signifying that the changes in mtDNA-CN and testosterone were correspondent during treatment with metformin. A literature search for this possible relationship found no human studies, but two murine studies were found where mtDNA-CN levels in skeletal muscles correlated with testosterone exposures [43, 44]. We found no impact from receiving a non-maximal regimen, but this could be due to the greater number of 
patients receiving a non-maximal regimen in normal weight patients. Compliance measure in this study also did not affect changes in mtDNA-CN or any of the other clinical factors. This is probably because patients returned to the clinic for prescriptions every 1 to 2 months, which ensured that there were continued use of medications, and very few pills were actually missed.

There are a few limitations to this study. Although we are currently the only group to demonstrate a change in mtDNA-CN during treatment with metformin in patients with PCOS, this study is a longitudinal cohort by design. A placebo-treated, age-matched control that matches the study group is needed to prove cause and effect, but we feel that this cannot be justified at this time due to a lack of previously reported interaction between metformin and mtDNA-CN. In addition, there is an ethical concern from withholding metformin when it has been proven effective in randomized control trials [45]. Secondly, although the cohort was identified using the Rotterdam criteria for PCOS [25], and we were able to identified a subset of patients with insulin resistance to explore its relationship to mtDNA-CN, our group is younger and had a glucose impaired rate of $14.8 \%$, compared with the $64.4 \%$ in a previous report [46]. This may affect the applicability of the study results to groups with more insulin resistance. Lastly, although 98.9\% (87/88) of patients returned at 6-month, there will inevitably be concerns about the lower number of patient remaining at 1-year $(61 / 88)$. It should be noted that the data at 1year was not used alone in statistical tests, but was part of hierarchical regression models (GEE). As the hierarchical regression model has been shown to be resistant to intermittently missing data [47], the results should remain valid as long as the data was missing at random. We checked this assumption by assessing for bias between patient characteristics, included anthropometric, hormonal, metabolic measures, factors such as nonmaximal regimen, medical compliance, and whether these affected patient remainingin the study at 1-year (See Supplementary Table 3, Additional file 1). None of the tested variables were related to whether patients remained in the study.

\section{Conclusion}

Time-dependent decreases in mtDNA-CN, 8-OHdG, testosterone, SHBG, and hepatic enzymes were seen in a longitudinal observational study in patients with PCOS, who received metformin for 1 year. The decrease in mtDNA-CN likely reflects an improvement in cellular oxidative stress, judging by the concurrent decrease in 8-OHdG. However, there is currently no causal research on the nature of these mtDNA-CN reductions. Clinicians should, therefore, remain cautious about the use of metformin as it does appear to alter mitochondria functions in these patients. We found no association between overweight, abdominal obesity, insulin resistance, patient compliance and the change in mtDNA-CN. Testosterone was correlated with the change in mtDNA$\mathrm{CN}$, and may have a role in modulating the effect of metformin on the mitochondria, but more work is needed to ascertain this relationship.

\section{Supplementary information}

Supplementary information accompanies this paper at https://doi.org/10. 1186/s12958-020-00629-5.

\section{Additional file 1 Supplementary Tables. Supplementary Table}

1. The normalized changes for each variable over the duration of the study. Supplementary Table $\mathbf{2}$. Coefficient estimates for medical compliance in explaining change in each clinical variable.

Supplementary Table 3. Coefficient estimates for each clinical variable in predicting patient drop-out from the logistic regression models.

\section{Abbreviations}

PCOS: Polycystic ovarian syndrome; mtDNA-CN: Mitochondria DNA copy number; 8-OHdG: 8-hydroxydeoxyguanosine; ROS: Reactive oxygen species; BMI: Body mass index; SBP: Systolic blood pressure; DBP: Diastolic blood pressure; GOT: Glutamic oxaloacetic transaminase; GPT: Glutamic pyruvic transaminase; hsCRP: High sensitivity C-reactive protein; FAl: Free androgen index; HOMA-IR: Homeostatic model assessment-insulin resistance; SHBG: Serum sex hormone-binding globulin; GEE: Generalized estimating equations

\section{Acknowledgements}

The authors acknowledge statistical assistance provided by the Center of Statistical Consultation and Research in the Department of Medical Research, National Taiwan University Hospital

\section{Authors' contributions}

Po-Kai Yang drafted the work and interpreted the data; Chia-Hong Chou made contributions in acquisition of the data; Chin-Hao Chang contributed to the analysis of the data; Shee-Uan Chen substantively revised the manuscript; Hong-Nerng Ho contributed to the conception of the study; Mei-Jou Chen contributed to the design of the work. The authors read and approved the final manuscript.

\section{Funding}

The study was supported by the Ministry of Science and Technology of Taiwan (MOST 105-2628-B002-043-MY4).

\section{Availability of data and materials}

The datasets used during the current study are available from the corresponding author on reasonable request.

Ethics approval and consent to participate

This study was approved by the Institutional Review Board of National Taiwan University Hospital.

Consent for publication

Not applicable.

\section{Competing interests}

The authors declare that they have no competing interests.

\section{Author details}

${ }^{1}$ Department of Obstetrics and Gynecology, National Taiwan University Hospital, No. 8, Chung-Shan South Road, 100 Taipei, Taiwan. ${ }^{2}$ College of Medicine, National Taiwan University, Taipei, Taiwan. ${ }^{3}$ Department of Medical Research, National Taiwan University Hospital, Taipei, Taiwan. ${ }^{4}$ College of Medicine, Taipei Medical University, Taipei, Taiwan. ${ }^{5}$ Livia Shangyu Wan Scholar, National Taiwan University, Taipei, Taiwan. 
Received: 12 May 2020 Accepted: 1 July 2020

\section{Published online: 13 July 2020}

\section{References}

1. Ehrmann DA. Medical progress: polycystic ovary syndrome. N Engl J Med. 2005;352(12):1223-36.

2. Murri M, Luque-Ramirez M, Insenser M, Ojeda-Ojeda M, Escobar-Morreale HF. Circulating markers of oxidative stress and polycystic ovary syndrome (PCOS): a systematic review and meta-analysis. Hum Reprod Update. 2013; 19(3):268-88.

3. Ilkun O, Boudina S. Cardiac dysfunction and oxidative stress in the metabolic syndrome: an update on antioxidant therapies. Curr Pharm Des. 2013;19(27):4806-17.

4. Macut D, Bjekic-Macut J, Savic-Radojevic A. Dyslipidemia and oxidative stress in PCOS. Front Horm Res. 2013;40:51-63.

5. Chen MJ, Chiu HM, Chen CL, Yang WS, Yang YS, Ho HN. Hyperandrogenemia is independently associated with elevated alanine aminotransferase activity in young women with polycystic ovary syndrome. J Clin Endocrinol Metab. 2010;95(7):3332-41.

6. Boland ML, Oldham S, Boland BB, Will S, Lapointe JM, Guionaud S, et al. Nonalcoholic steatohepatitis severity is defined by a failure in compensatory antioxidant capacity in the setting of mitochondrial dysfunction. World J Gastroenterol. 2018;24(16):1748-65.

7. Victor VM, Rocha M, Banuls C, Sanchez-Serrano M, Sola E, Gomez M, et al. Mitochondrial complex I impairment in leukocytes from polycystic ovary syndrome patients with insulin resistance. J Clin Endocrinol Metab. 2009; 94(9):3505-12

8. Victor VM, Rovira-Llopis S, Banuls C, Diaz-Morales N, Castello R, Falcon R, et al. Effects of metformin on mitochondrial function of leukocytes from polycystic ovary syndrome patients with insulin resistance. Eur J Endocrinol. 2015;173(5):683-91.

9. Lee S-H, Chung D-J, Lee H-S, Kim T-J, Kim M-H, Jeong HJ, et al. Mitochondrial DNA copy number in peripheral blood in polycystic ovary syndrome. Metabolism. 2011;60(12):1677-82

10. Lee $\mathrm{HC}$, Yin $\mathrm{PH}$, Chi CW, Wei $\mathrm{YH}$. Increase in mitochondrial mass in human fibroblasts under oxidative stress and during replicative cell senescence. J Biomed Sci. 2002:9(6):517-26.

11. Michel S, Wanet A, De Pauw A, Rommelaere G, Arnould T, Renard P. Crosstalk between mitochondrial (dys) function and mitochondrial abundance. J Cell Physiol. 2012;227(6):2297-310.

12. Clay Montier LL, Deng JJ, Bai Y. Number matters: control of mammalian mitochondrial DNA copy number. J Genet Genomics. 2009;36(3):125-31.

13. Al-Kafaji G, Bakheit HF, Alharbi MA, Farahat AA, Jailani M, Ebrahin BH, et al. Mitochondrial DNA copy number in peripheral blood as a potential non-invasive biomarker for multiple sclerosis. Neuromolecular Med. 2020;22:304-13.

14. Pyle A, Anugrha H, Kurzawa-Akanbi M, Yarnall A, Burn D, Hudson G. Reduced mitochondrial DNA copy number is a biomarker of Parkinson's disease. Neurobiol Aging. 2016;38:216.e7-e10.

15. Velazquez EM, Mendoza S, Hamer T, Sosa F, Glueck CJ. Metformin therapy in polycystic ovary syndrome reduces hyperinsulinemia, insulin resistance, hyperandrogenemia, and systolic blood pressure, while facilitating normal menses and pregnancy. Metabolism. 1994;43(5):647-54.

16. Nestler JE, Jakubowicz DJ. Decreases in ovarian cytochrome P450c17 alpha activity and serum free testosterone after reduction of insulin secretion in polycystic ovary syndrome. N Engl J Med. 1996;335(9):617-23.

17. Yang PK, Hsu CY, Chen MJ, Lai MY, Li ZR, Chen CH, et al. The efficacy of 24month metformin for improving menses, hormones, and metabolic profiles in polycystic ovary syndrome. J Clin Endocrinol Metab. 2018;103(3):890-9.

18. Cameron AR, Logie L, Patel K, Erhardt S, Bacon S, Middleton P, et al. Metformin selectively targets redox control of complex I energy transduction. Redox Biol. 2018;14:187-97.

19. Hattori Y, Suzuki K, Hattori S, Kasai K. Metformin inhibits cytokine-induced nuclear factor kappaB activation via AMP-activated protein kinase activation in vascular endothelial cells. Hypertension. 2006;47(6):1183-8.

20. Bergheim I, Luyendyk JP, Steele C, Russell GK, Guo L, Roth RA, et al. Metformin prevents endotoxin-induced liver injury after partial hepatectomy. J Pharmacol Exp Ther. 2006;316(3):1053-61.

21. Kocer D, Bayram F, Diri H. The effects of metformin on endothelial dysfunction, lipid metabolism and oxidative stress in women with polycystic ovary syndrome. Gynecol Endocrinol. 2014;30(5):367-71.
22. Piomboni P, Focarelli R, Capaldo A, Stendardi A, Cappelli V, Cianci A, et al. Protein modification as oxidative stress marker in follicular fluid from women with polycystic ovary syndrome: the effect of inositol and metformin. J Assist Reprod Genet. 2014;31(10):1269-76.

23. Sova H, Puistola U, Morin-Papunen L, Karihtala P. Metformin decreases serum 8-hydroxy-2'-deoxyguanosine levels in polycystic ovary syndrome. Fertil Steril. 2013;99(2):593-8.

24. Valavanidis A, Vlachogianni T, Fiotakis C. 8-hydroxy-2' -deoxyguanosine (8-OHdG): a critical biomarker of oxidative stress and carcinogenesis. J Environ Sci Health C Environ Carcinog Ecotoxicol Rev. 2009;27(2):12039.

25. Revised 2003 consensus on diagnostic criteria and long-term health risks related to polycystic ovary syndrome. Fertil Steril. 2004;81(1):19-25.

26. WHO Expert Consultation. Appropriate body-mass index for Asian populations and its implications for policy and intervention strategies. Lancet. 2004;363(9403):157-63.

27. World Health Organization. The Asia-Pacific perspective: redefining obesity and its treatment. Health Communications Australia. 2000.

28. Chen MJ, Yang WS, Chen CL, Wu MY, Yang YS, Ho HN. The relationship between anti-Mullerian hormone, androgen and insulin resistance on the number of antral follicles in women with polycystic ovary syndrome. Hum Reprod. 2008;23(4):952-7.

29. American Diabetes Association. 2. Classification and diagnosis of diabetes: standards of medical Care in Diabetes-2020. Diabetes Care. 2020; 43(Supplement 1):S14-31.

30. Matthews DR, Hosker JP, Rudenski AS, Naylor BA, Treacher DF, Turner RC. Homeostasis model assessment: insulin resistance and beta-cell function from fasting plasma glucose and insulin concentrations in man. Diabetologia. 1985;28(7):412-9.

31. Chang YK, Kim DE, Cho SH, Kim JH. Association between leukocyte mitochondrial DNA copy number and regular exercise in postmenopausal women. Korean J Fam Med. 2016;37(6):334-9.

32. Liang K-Y, Zeger SL. Longitudinal data analysis using generalized linear models. Biometrika. 1986;73(1):13-22.

33. Bai RK, Wong LJ. Simultaneous detection and quantification of mitochondrial DNA deletion(s), depletion, and over-replication in patients with mitochondrial disease. J Mol Diagn. 2005;7(5):613-22.

34. Gu F, Chauhan V, Kaur K, Brown WT, LaFauci G, Wegiel J, et al. Alterations in mitochondrial DNA copy number and the activities of electron transport chain complexes and pyruvate dehydrogenase in the frontal cortex from subjects with autism. Transl Psychiatry. 2013;3:e299.

35. Qiu C, Hevner K, Abetew D, Sedensky M, Morgan P, Enquobahrie DA, et al. Mitochondrial DNA copy number and oxidative DNA damage in placental tissues from gestational diabetes and control pregnancies: a pilot study. Clin Lab. 2013;59(5-6):655-60.

36. Liu CS, Tsai CS, Kuo CL, Chen HW, Lii CK, Ma YS, et al. Oxidative stressrelated alteration of the copy number of mitochondrial DNA in human leukocytes. Free Radic Res. 2003;37(12):1307-17.

37. Lin CS, Wang LS, Tsai CM, Wei YH. Low copy number and low oxidative damage of mitochondrial DNA are associated with tumor progression in lung cancer tissues after neoadjuvant chemotherapy. Interact Cardiovasc Thorac Surg. 2008;7(6):954-8.

38. Palmeira CM, Serrano J, Kuehl DW, Wallace KB. Preferential oxidation of cardiac mitochondrial DNA following acute intoxication with doxorubicin. Biochim Biophys Acta-Bioenerg. 1997;1321(2):101-6.

39. Sova H, Morin-Papunen L, Puistola U, Karihtala P. Distinctively low levels of serum 8-hydroxydeoxyguanosine in women with polycystic ovary syndrome. Fertil Steril. 2010;94(7):2670-3.

40. Abe $Y$, Kikuchi T, Nagasaki K, Hiura M, Tanaka Y, Ogawa Y, et al. Usefulness of GPT for diagnosis of metabolic syndrome in obese Japanese children. J Atheroscler Thromb. 2009;16(6):902-9.

41. Black CN, Bot M, Scheffer PG, Penninx BW. Sociodemographic and lifestyle determinants of plasma oxidative stress markers 8-OHdG and F2Isoprostanes and associations with metabolic syndrome. Oxid Med Cell Longev. 2016;2016:7530820.

42. Zhang J, Bao Y, Zhou X, Zheng L. Polycystic ovary syndrome and mitochondrial dysfunction. Reprod Biol Endocrinol. 2019;17(1):67.

43. Guo W, Wong S, Li M, Liang W, Liesa M, Serra C, et al. Testosterone plus low-intensity physical training in late life improves functional performance, skeletal muscle mitochondrial biogenesis, and mitochondrial quality control in male mice. PLoS One. 2012;7(12):e51180. 
44. Liu C, Ma J, Zhang J, Zhao H, Zhu Y, Qi J, et al. Testosterone deficiency caused by castration modulates mitochondrial biogenesis through the AR/ PGC1a/TFAM pathway. Front Genet. 2019;10:505.

45. Teede HJ, Misso ML, Costello MF, Dokras A, Laven J, Moran L, et al. Recommendations from the international evidence-based guideline for the assessment and management of polycystic ovary syndrome. Hum Reprod. 2018;33(9):1602-18.

46. DeUgarte CM, Bartolucci AA, Azziz R. Prevalence of insulin resistance in the polycystic ovary syndrome using the homeostasis model assessment. Fertil Steril. 2005;83(5):1454-60.

47. Twisk J, de Vente W. Attrition in longitudinal studies. How to deal with missing data. J Clin Epidemiol. 2002;55(4):329-37.

\section{Publisher's Note}

Springer Nature remains neutral with regard to jurisdictional claims in published maps and institutional affiliations.

Ready to submit your research? Choose BMC and benefit from:

- fast, convenient online submission

- thorough peer review by experienced researchers in your field

- rapid publication on acceptance

- support for research data, including large and complex data types

- gold Open Access which fosters wider collaboration and increased citations

- maximum visibility for your research: over $100 \mathrm{M}$ website views per year

At BMC, research is always in progress.

Learn more biomedcentral.com/submissions 